\title{
An Emergency Medicine Approach to Neonatal Hyperbilirubinemia
}

\author{
James E. Colletti, MD, FAAEM, FAAP ${ }^{a, *}$, \\ Samip Kothori, $\mathrm{MD}^{\mathrm{b}}$, Danielle M. Jackson, $\mathrm{MD}^{\mathrm{c}}$, \\ Kevin P. Kilgore, $\mathrm{MD}^{\mathrm{c}}$, Kelly Barringer, $\mathrm{MD}^{\mathrm{c}}$ \\ ${ }^{a}$ Department of Emergency Medicine, Mayo Clinic College of Medicine, \\ 200 First St. SW, Rochester, MN 55905, USA \\ ${ }^{\mathrm{b}}$ Department of Pediatrics, University of Arizona, \\ 150 N. Campbell Avenue, Tucson, AZ 85724, USA \\ ${ }^{\mathrm{c}}$ Department of Emergency Medicine, Regions Hospital, 640 Jackson Street, \\ Mail Stop 11102F, St. Paul, MN 55101, USA
}

Jaundice, also known as hyperbilirubinemia, is a yellowish-greenish pigmentation of the sclera and skin caused by an increase in bilirubin production or a defect in bilirubin elimination. Jaundice is defined by a serum bilirubin concentration greater than $5 \mathrm{mg} / \mathrm{dL}$. Neonatal jaundice is estimated to occur in the majority of term infants $(60 \%)$ in the first week of life, and approximately $2 \%$ of infants reach total serum bilirubin (TSB) levels in excess of $20 \mathrm{mg} / \mathrm{dL}$ [1-4]. The TSB normally rises during the first 3 to 5 days of life and then begins to decline [3]. As such, it is important that bilirubin levels are interpreted based on the neonate's age in hours [5].

The feared complication of neonatal jaundice is bilirubin encephalopathy, the result of prolonged unconjugated hyperbilirubinemia. Acute bilirubin encephalopathy can eventually develop into chronic bilirubin encephalopathy (kernicterus). Kernicterus has been called the ultimate adverse manifestation of severe hyperbilirubinemia [6] and was rarely seen in the decades following the introduction of phototherapy and exchange transfusion; however, recent reports suggest it is reemerging [6]. A concerning number of cases have been reported in healthy term and near-term neonates [7]. Since 1990, the Pilot Kernicterus Registry has identified kernicterus in greater than 120 near-term and term infants who had been discharged as healthy from the hospital [7-10]. In 2001, the Joint Commission on Accreditation of Health Care Organizations issued a sentinel event alert notifying

\footnotetext{
* Corresponding author.

E-mail address: jamesecolletti@gmail.com (J.E. Colletti).
} 
health care providers and hospitals that kernicterus is a threat to otherwise healthy newborns. The reemergence of kernicterus has been attributed in part to earlier hospital discharge, before the natural peak of bilirubin in the neonate, as well as a result of relaxation of treatment criteria $[4,9,11,12]$.

Newborns are often discharged from the hospital within 48 hours of birth; as a result, hyperbilirubinemia is not as often detected before discharge as it had been previously [13]. The practice of early newborn discharge has transformed neonatal hyperbilirubinemia from an inpatient issue to an outpatient one $[14,15]$. Currently, hyperbilirubinemia is one of the most common reasons for readmission of a newborn [16,17]. As such, emergency physicians should be comfortable with the diagnosis, evaluation, and management of the jaundiced newborn [2,18]. Vales eloquently stated in a commentary that, in an ideal world, "the issue of dangerous hyperbilirubinemia should have been solved before embarking in a drastic cut on the in-hospital observation" of newborns [19].

\section{Epidemiology}

Hyperbilirubinemia is one of the most common reasons for the presentation of neonates to the emergency department and one of the major causes for hospital readmission. Overall, jaundice is observed in the first week of life in $60 \%$ of term infants and $80 \%$ of preterm infants $[1,20]$. In a casecontrol review, Maisels and Kring [17] concluded that hyperbilirubinemia is the major reason for hospital readmission in the first 2 weeks of life (incidence of 4.2 cases per 1000 discharges). Although the percentage of jaundiced newborns is higher among preterm newborns, the majority of data on the assessment, evaluation, and management of jaundice have been collected on term newborns with birth weights of $2500 \mathrm{~g}$ or greater.

\section{Pathophysiology}

Bilirubin is produced from the breakdown of hemoglobin (Fig. 1). Hemoglobin is degraded by heme oxygenase, resulting in the release of iron and the formation of carbon monoxide and biliverdin. Biliverdin is then converted to bilirubin by biliverdin reductase.

Unconjugated bilirubin (also known as indirect bilirubin) is initially only soluble in lipids, not water, and is subsequently bound by albumin in the blood stream. Any substance competing for binding sites, such as organic acids or drugs, can increase the levels of free bilirubin. In this unconjugated state, bilirubin is difficult to excrete (because it is lipid soluble), and it can easily pass into the central nervous system where it is neurotoxic and can produce kernicterus [11]. Unconjugated bilirubin is taken up by the liver, where it is conjugated by uridine diphosphate glucuronosyltransferase (UDPGT) to a conjugated form. 


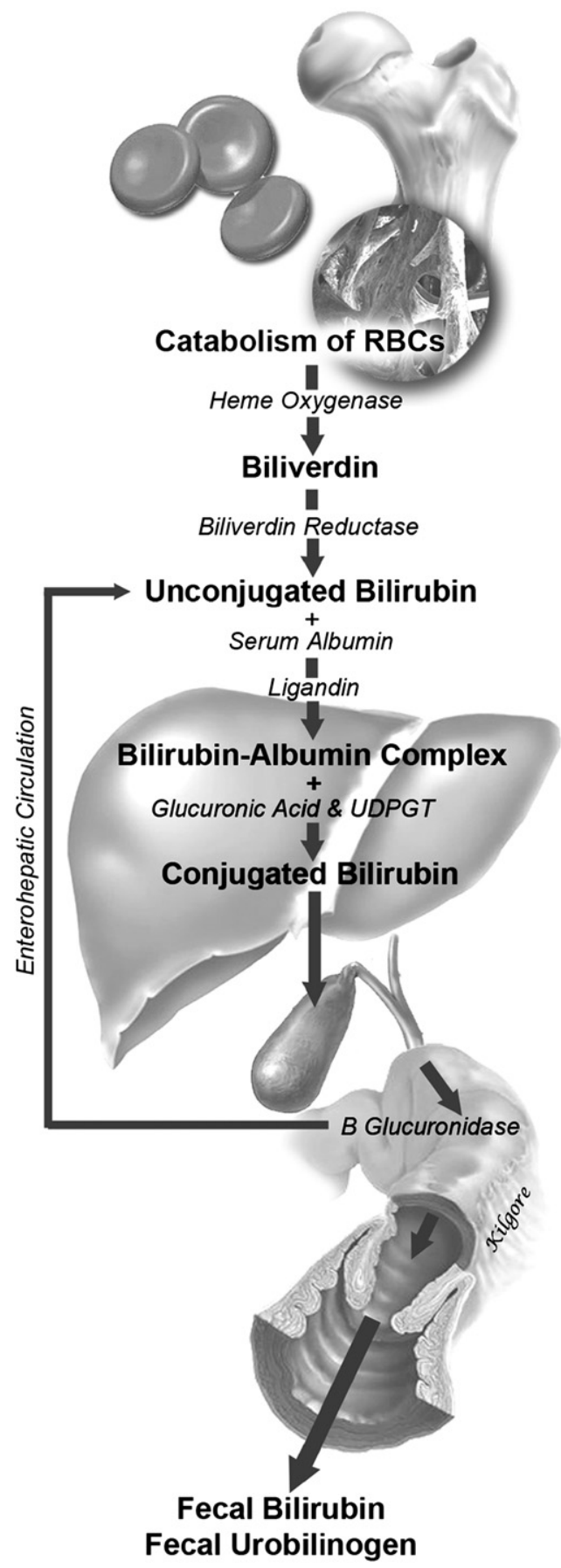

Fig. 1. The pathophysiology of neonatal hyperbilirubinemia. 
Conjugated bilirubin (direct bilirubin) is water soluble, non-toxic, and unable to cross the blood-brain barrier. It also binds to albumin and can compete with unconjugated bilirubin for binding sites. It is excretable into the biliary or intestinal tract. Once conjugated bilirubin enters the intestinal tract, it is either excreted in stool or deconjugated by bacteria, where it may reenter the circulation (enterohepatic circulation). Total bilirubin is bound to protein (mainly albumin) in the blood and is a combination of unconjugated and conjugated bilirubin.

There are three main causes of hyperbilirubinemia in the neonate, which generally manifests as elevated levels of unconjugated bilirubin: (1) an increase in hemolysis, (2) a delay in maturation or inhibition of conjugating mechanisms in the liver, and (3) interference with hepatic uptake of unconjugated bilirubin. Neonates are especially prone to hyperbilirubinemia secondary to increased hemoglobin from high red blood cell volume, shortened red blood cell survival (and therefore increased breakdown), and relative immaturity of UDPGT in the liver.

\section{Clinical presentation}

The emergency physician should be familiar with historical clues that put the neonate at increased risk for severe hyperbilirubinemia. Risk factors to consider are jaundice in the first 24 hours, visible jaundice before hospital discharge, fetal-maternal blood type incompatibility (ABO incompatibility as well as $\mathrm{Rh}$ incompatibility), prematurity, exclusive breastfeeding as well as significant weight loss associated with breastfeeding, maternal age greater than or equal to 25 years, male sex, delayed meconium passage, and excessive birth trauma such as bruising or cephalohematomas $[6,21,22]$. The parents should be asked specifically about poor feeding, urine output (including dark urine), stooling (delayed passage of meconium or light-colored stool), vomiting, or any changes in behavior (lethargy, changes in cry pattern, cries becoming more shrill, arching of the body) [18].

Pertinent family history includes ethnicity, as well as siblings with hyperbilirubinemia, anemia, liver disease, or splenectomy. Ethnicity is a factor in determining the likelihood of hyperbilirubinemia. Individuals of East Asian descent, certain tribes of Native Americans such as the Navajo, and those of Greek ancestry have a higher incidence of hyperbilirubinemia $[3,15,23]$. It is important to inquire about siblings with jaundice, because there is a 12.5 times greater risk for severe jaundice in newborns who have one or more siblings affected with severe neonatal hyperbilirubinemia when compared with newborns who have prior siblings without severe neonatal hyperbilirubinemia [24].

Physical assessment begins with the clinical appearance of the infant. Jaundice is assessed through blanching the skin with digital pressure, revealing the underlying color of the skin and subcutaneous tissue. The clinical 
assessment of jaundice is best undertaken in a well-lit room to maximize the ability to determine true skin color [21]. Jaundice in term and preterm infants follows a cephalocaudal progression [25]. Visual estimation of the severity of jaundice is unpredictably and imprecisely related to the actual serum bilirubin level, especially in infants with dark skin pigmentation and once the jaundice has extended to the lower legs and feet [5,9,26-28]. Moyer and colleagues [28] evaluated 122 healthy infants who underwent examination by two observers followed by measurements of serum bilirubin. The investigation concluded that visual estimation of infantile jaundice was not reliable, and that prediction of serum bilirubin concentration by clinical examination is not accurate [28]. After assessing the skin, it is important to look for other signs indicative of pathologic jaundice, such as pallor, petechiae, hydration, and weight status. Signs of blood loss or blood sequestration such as excessive bruising, hepatosplenomegaly, or cephalohematoma should be sought out as well [22].

A review of the birth history should be performed. First, it is important to establish whether the child was large for gestational age (LGA), within normal range, or small for gestational age (SGA). Infants that are SGA have associated complications such as hypoglycemia, polycythemia, and abnormal neurologic symptoms. Although hyperbilirubinemia is not directly linked to SGA infants, polycythemia can lead to an increased bilirubin level secondary to increased red cell destruction [29,30]. Infants that are LGA have an increased incidence of birth trauma due to their size. Excessive bruising and cephalohematomas can be acquired from birth trauma. One study determined that over a 10-year period, $2.5 \%$ of all breech births had an associated cephalohematoma [31]. Forceps and vacuum extraction deliveries can also cause bruising and cephalohematomas to occur [30]. Other injuries such as clavicle fractures and brachial plexus injuries are also associated with hyperbilirubinemia. Clavicle fractures are often secondary to shoulder dystocia. These injuries are linked to hyperbilirubinemia secondary to the extravasation of blood from the associated trauma [32]. Information regarding medications administered to the mother before and during delivery should be obtained, because certain medications, such as oxytocin, can result in an increased incidence of hyperbilirubinemia [30,33].

On initial inspection of a jaundiced neonate, the clinician should have an increased index of suspicion for underlying disease entities. Among these entities are sepsis and galactosemia. A diagnosis of sepsis should be considered in the infant presenting with apnea or temperature instability. Galactosemia presents as feeding intolerance manifested by persistent vomiting, an enlarged liver, seizures, and lethargy [34].

\section{Differential diagnosis}

There are two different classifications of jaundice to considerphysiologic and pathologic. Physiologic jaundice is the transient elevation 
of serum bilirubin during the first week of life. Pathologic jaundice occurs in the first 24 hours of life and is often associated with anemia or hepatosplenomegaly. Furthermore, pathologic jaundice can be characterized by a rapidly rising serum bilirubin ( $>5 \mathrm{mg} / \mathrm{dL}$ per day), prolonged jaundice ( $>7$ to 10 days in a full-term infant), and an elevated conjugated bilirubin concentration $(>2 \mathrm{mg} / \mathrm{dL}$ or more than $20 \%$ of TSB).

Two main categories of hyperbilirubinemia are unconjugated or conjugated, also known, respectively, as indirect or direct. One may further breakdown the etiology of unconjugated hyperbilirubinemia into an increased bilirubin load, decreased bilirubin excretion, or bilirubin increase due to breastfeeding. One of the major causes of increased bilirubin load is blood group incompatibility.

ABO blood group incompatibility is the leading cause of hemolytic disease of the newborn. One of the primary clinical features of ABO blood group incompatibility is hyperbilirubinemia. Approximately one third of infants with $\mathrm{ABO}$ incompatibility have a positive direct antiglobulin test (DAT or Coombs' test), indicating that they have anti-A or anti-B antibodies attached to their red cells [35]. Of this third, 20\% will have a peak TSB of greater than $12.8 \mathrm{mg} / \mathrm{dL}$ [35]. Although ABO-incompatible, DATpositive infants are more likely to develop moderate hyperbilirubinemia, severe jaundice is uncommon [35]. Overall, ABO hemolytic disease is a common cause of early hyperbilirubinemia before the infant leaves the nursery; it is a rare cause of hyperbilirubinemia in infants who are discharged and return for evaluation [35]. A mechanism similar to that of ABO incompatibility exists for Rh incompatibility. Of note, $15 \%$ of all births are a set-up for $\mathrm{ABO}$ incompatibility, but only $0.33 \%$ to $2.2 \%$ of all neonates will have some manifestation of the disease [36,37]. Several other minor blood group types, such as Kell and Duffy, can also cause hemolytic disease.

Enzyme deficiencies in the glycolysis pathway, such as glucose-6-phosphate dehydrogenase (G6PD) deficiency and pyruvate kinase deficiency, also increase the indirect bilirubin load. G6PD deficiency mainly affects the red blood cells and can cause acute hemolysis. Many infants, although not all, with this disease present with hyperbilirubinemia. The exact mechanism as to how G6PD deficiency causes hyperbilirubinemia is unknown; however, the leading explanation is a decrease in bilirubin elimination [38]. G6PD deficiency affects between 200 and 400 million people worldwide and is the leading cause of hyperbilirubinemia in certain population subtypes such as African Americans, those of Mediterranean descent, and some Far East populations [39,40]. Multiple studies have shown that infants who are affected by hyperbilirubinemia and who have G6PD deficiency have the same clinical course and clinical indicators as infants with hyperbilirubinemia who do not have G6PD deficiency; therefore, it is prudent to include G6PD deficiency in the differential diagnosis if there is a family history of G6PD deficiency or if the patient fits into an at-risk demographic group $[39,41]$. 
Pyruvate kinase deficiency is the second leading cause of red blood cell enzyme deficiency in North America and overall is an uncommon cause of hyperbilirubinemia. The clinician should have a higher index of suspicion for pyruvate kinase deficiency as a cause of hyperbilirubinemia in populations in which pyruvate kinase deficiency is more common, such as neonates of Indian descent $[42,43]$. An investigation by Kedar and colleagues determined the prevalence of pyruvate kinase deficiency in jaundiced neonates in India to be $3.21 \%$ [43].

Abnormalities in the red blood cell membrane, such as hereditary spherocytosis and elliptocytosis/ovalocytosis, may also result in an increase in indirect bilirubin levels. In hereditary spherocytosis, there is a defect in the red cell membrane causing an increase in red cell breakdown and abnormal red blood cells. Fifty percent of individuals with hereditary spherocytosis state they had significant jaundice as newborns. Trucco and Brown [44] suggest that the clinical findings of hereditary spherocytosis can include early onset of jaundice or a marked increase in bilirubin beyond what is expected with physiologic jaundice. An osmotic fragility test is considered to be the gold standard to confirm a diagnosis of hereditary spherocytosis $[45,46]$. Elliptocytosis/ovalocytosis is an autosomal dominant disease affecting predominantly Southeast Asian and Mediterranean populations. Several case studies have reported that it results in an elevation of indirect bilirubin $[47,48]$.

It is important to exclude hemolytic disease as the cause of increased indirect bilirubin levels. The diseases discussed previously can cause hemolysis, and it is important to identify factors that will help diagnosis hemolytic disease early. A family history of significant hemolytic disease, a high-risk ethnic background, early or severe jaundice, or the development of jaundice in the newborn before 24 hours of life suggest the possibility of hemolytic disease in the newborn. In addition, a bilirubin level in a neonate that does not respond to phototherapy may indicate that breakdown is ongoing from an underlying disease process that requires further evaluation. A rapid rate of rise in the bilirubin level should cause concern for hemolytic disease $[1,13]$.

Increased indirect bilirubin can also be caused by decreased bilirubin excretion. One of the main causes of decreased bilirubin excretion is that neonates have a decrease in bowel motility which results in an increase in the enterohepatic circulation, in turn, leading to increased indirect bilirubin $[22,49]$. One of the more life-threatening causes of hyperbilirubinemia by impaired bilirubin excretion is Crigler-Najjar syndrome. There are two types, both of which are due to defects in bilirubin-UDP-glucuronosyltransferase (B-UGT). Type I is characterized by an absence of enzyme activity and is the more severe type which can lead to kernicterus and death by the age of 1 to 2 years of age without treatment. Type II has some enzyme activity and is therefore less fatal. Without B-UGT, unconjugated bilirubin accumulates in the body. Once the threshold of albumin and other tissue 
phospholipids is reached, the brain becomes stained and kernicterus develops. The disease is rare and inherited in an autosomal recessive fashion. The clinical presentation includes elevated bilirubin levels and symptoms of kernicterus despite phototherapy treatment [50,51]. Congenital hypothyroidism is another disease that can cause hyperbilirubinemia through decreased bilirubin excretion. It presents with prolonged jaundice, lethargy, constipation, poor feeding, hypotonia, and enlarged fontanelles. It is extremely important to follow-up on thyroid levels in the newborn genetic screening of neonates who have hyperbilirubinemia with concerns of hypothyroidism [52].

Increased indirect bilirubin levels can also result from breastfeeding. When compared with formula feeding, breastfeeding has been associated with neonatal jaundice that is more severe and longer in duration $[53,54]$. This type of hyperbilirubinemia can present in two ways. The first is a delayed course in which the bilirubin level rises on the fourth to seventh day of life after physiologic jaundice is resolving. The bilirubin then peaks at about 2 weeks of life (to a level of $15-25 \mathrm{mg} / \mathrm{dL}$ ) and stays in this range for another 2 weeks before a decrease is observed [55]. The second type has also been described as an exaggerated physiologic jaundice. It occurs earlier than the first type, typically on the third or fourth day of life, with bilirubin levels above $10 \mathrm{mg} / \mathrm{dL}$ [54]. The exact mechanism of action is unknown. There is evidence to suggest that caloric deprivation results in hyperbilirubinemia. Because it may take anywhere from 2 to 5 days for the production of breast milk, a breastfed infant may experience a calorie deficiency [56]. Furthermore, it has been shown that human breast milk has a substance within it that inhibits hepatic B-UGT, which, in turn, leads to hyperbilirubinemia [57,58]. It is recommended by the American Academy of Pediatrics that, even with this risk, it is still better to breastfeed than to switch to formula when breastfeeding jaundice is suspected [1]. Human milk jaundice syndrome, also know as breastfeeding-associated jaundice, should be distinguished from breastfeeding jaundice. Human milk jaundice appears later than breastfeeding jaundice, typically on the fourth to seventh day of life, and is more prolonged than breastfeeding jaundice [35].

Important clues to the etiology of jaundice are the time of onset and the duration [59,60]. Jaundice in the first 24 hours may be secondary to blood type incompatibilities such as ABO and Rh. Sepsis should also be considered, because several investigations have described as association between proven bacterial infection and neonatal jaundice [60-66]. Arthur and Williams [62], Seeler [65], and Littlewood [64] demonstrated an association between hyperbilirubinemia and urinary tract infections in neonates. A few investigations have concluded that jaundice as the only symptom of sepsis is rare, especially in a well-appearing neonates [67-69].

Jaundice appearing during the second to third day of life is most likely physiologic and will dissipate by the fifth or sixth day. If the newborn is breastfed, he or she may develop breastfeeding jaundice that can be present 
for 2 weeks. The clinician should consider a pathologic etiology of jaundice if it persists for longer than 2 weeks. A prospective investigation by Maisels and Kring [69] demonstrated that most newborns admitted for indirect hyperbilirubinemia are healthy breastfed infants. Newborns who warrant careful screening are those presenting with late-onset jaundice, direct hyperbilirubinemia, or signs of sepsis.

Conjugated hyperbilirubinemia is concerning because, although it is nontoxic, it is a marker for serious underlying disease. Etiologies of conjugated hyperbilirubinemia are summarized in Table 1. Associated findings of conjugated hyperbilirubinemia are pale acholic stools, dark urine, or the presence of bilirubin in the urine [1].

\section{Emergency department evaluation}

Evaluation should be guided by the clinical appearance of the neonate and the timing of jaundice. A full-term, well-appearing, asymptomatic infant is at low risk of complications, especially in the absence of danger signs (Box 1) and when presenting within a time frame that is consistent with physiologic jaundice [70]. To aid in the assessment of the jaundiced neonate, several laboratory investigations may be obtained, including TSB, a direct Coombs' test, blood group testing, hemoglobin, urinalysis, as well as a full sepsis work-up (white blood cell count, cerebral spinal fluid for analysis and culture, and blood and urine cultures).

A serum bilirubin and hemoglobin measurement should be routinely ordered for the jaundiced infant. Inaccuracy between the physician's clinical estimation and actual serum bilirubin levels is well documented, as is poor interrater reliability; therefore, physical examination alone should not be relied upon. Measurements of total and direct serum bilirubin should be obtained to differentiate conjugated from unconjugated hyperbilirubinemia. Unfortunately, measurement of bilirubin has a notorious wide range, is inaccurate, and is associated with a tremendous amount of interlaboratory variability [70-78]. In a well-appearing, afebrile infant with unconjugated hyperbilirubinemia and normal hemoglobin, no further tests are needed (although some practitioners would obtain a urinalysis and urine culture) [2].

Table 1

Etiology of conjugated hyperbilirubinemia

\begin{tabular}{lll}
\hline Infectious & Anatomic & Metabolic \\
\hline TORCH & Biliary atresia & Inborn errors of metabolism \\
Hepatitis & Choledochal cyst & Cystic fibrosis \\
Sepsis (usually presents with & Alagille syndrome & Alpha-1-antitrypsin deficiency \\
other signs of sepsis, ie, & (arteriohepatic & \\
vomiting, abdominal & dysplasia) & \\
distention, respiratory & Cholestasis & \\
distress, and poor feeding) & Mass lesion & \\
\hline
\end{tabular}




\section{Box 1. Danger signs}

1. Family history of significant hemolytic disease

2. Vomiting

3. Lethargy

4. Poor feeding

5. Fever

6. Onset of jaundice after the third day of life

7. High-pitched cry

8. Dark urine

9. Light stools

As alluded to earlier in this manuscript, obtaining a urinalysis may prove helpful in the infant with physiologic jaundice. Several investigations have indicated an association between jaundice and asymptomatic urinary tract infections $[2,62,64,67,79-82]$. A series of articles by Seeler indicate that jaundice may be one of the earliest signs of a urinary tract infection [64,79,81]. $\mathrm{Ng}$ and Rawstron described a case series of six neonates in whom jaundice was the prominent feature in acute urinary tract infection [80]. Chavalitdhamrong and colleagues [68] prospectively identified two urinary tract infections in 69 infants with unexplained jaundice. Rooney and colleagues [62] found urinary tract infections in $40 \%$ (9 of 22) of infants with documented bacterial infections. Garcia and Nager [82] prospectively evaluated asymptomatic, afebrile, jaundiced infants for evidence of a urinary tract infection, which they found in $7.5 \%$ of infants less than 8 weeks of age. Furthermore, neonates presenting with an onset of jaundice after 8 days of age or those with an elevated conjugated bilirubin fraction were more likely to have a urinary tract infection. Garcia and Nager recommend testing for a urinary tract infection as part of the evaluation of the asymptomatic jaundiced infant presenting to the emergency department [82].

In cases in which anemia is present, a complete blood count with peripheral smear, Coombs' test, and maternal and fetal blood types should be analyzed (ABO and $\mathrm{Rh}(\mathrm{D})$ typing) [2]. Neonates who are Coombs' positive are approximately twice as likely as their comparable peers to have a serum bilirubin level of more than $12 \mathrm{mg} / \mathrm{dL}$ [70]. In the presence of a positive Coombs' test, $\mathrm{ABO}$ or $\mathrm{Rh}$ incompatibility is the most likely cause of jaundice, because the majority of neonates with other causes of hemolysis will be Coombs' negative [2]. Furthermore, a G6PD level has been recommended for any infant undergoing phototherapy with an appropriate genetic or geographic background, and for any neonate who does not respond well to phototherapy [2].

In cases of conjugated hyperbilirubinemia, evaluation efforts should be directed to determining the underlying etiology [2]. Most commonly, 
conjugated hyperbilirubinemia is infectious in origin, and these causes should be assessed with a TORCH infection panel (toxoplasmosis, others, rubella, cytomegalovirus, and herpes simplex), hepatitis B serology, and urinalysis for cytomegalovirus. Although less common, jaundice may be the presenting complaint in neonates with inborn errors of metabolism, cystic fibrosis, alpha-1-antitrypsin deficiency, and iron storage deficiencies. When any of these processes are suspected, investigative studies should be broad and include liver function tests, ammonia, albumin and total protein levels, and a complete chemistry panel. Examination of urine for reducing substances, sweat chloride testing, and red blood cell galactose-1-phosphate uridyltransferase activity may be required.

Obstructive causes may be more difficult to identify owing to their insidious and often intermittent presentation. Abdominal ultrasound and hepatobiliary scintigraphy to assess for biliary atresia or a choledochal cyst may provide a diagnosis that, when reached promptly, improves outcomes due to early surgical intervention.

In cases of significant hyperbilirubinemia (serum bilirubin level $>15 \mathrm{mg} / \mathrm{dL}$ ) with or without symptoms of illness, the following laboratory values should be obtained: direct and indirect bilirubin, complete blood cell count, peripheral blood smear for hemolysis, reticulocyte count, liver function tests, thyroid function tests, as well as an evaluation for sepsis. If an exchange transfusion is anticipated, a type and cross must be obtained. Indirect hyperbilirubinemia, reticulocytosis, and a smear consistent with red blood cell destruction are suggestive of hemolysis [58]. Infants with hemolysis as a cause of their hyperbilirubinemia are at greater risk for kernicterus [83] and should be admitted.

\section{Kernicterus}

Kernicterus (bilirubin encephalopathy) is a rare but catastrophic bilirubin-induced brain injury that is one of the known causes of cerebral palsy $[7,35,84,85]$. It is one of the few causes of brain damage in the neonatal period that, with current diagnostic and treatment regimens, is preventable [11]. The link between hyperbilirubinemia and brain damage was first established in the early 1950s when Mollison and Hsia and colleagues demonstrated that the risk of kernicterus in infants with hemolytic disease of the newborn dramatically increased with the bilirubin level and that an exchange transfusion could dramatically decrease that risk [70,86,87]. The likelihood of kernicterus depends on the bilirubin level, as well as the age of the child and comorbidities.

A schema for grading the severity of acute bilirubin encephalopathy has been described $[10,88]$. The earliest signs are often subtle and may be missed but include early alterations in tone of the extensor muscles (hypotonia or hypertonia), retrocollis (backward arching of the neck), opisthotonus 
(backward arching of the trunk), as well as a poor suck response $[10,13,89]$. The hypertonia and retrocollis will often increase in severity and may be by accompanied by a shrill cry as well as unexplained irritability alternating with lethargy and fever [89]. Prompt and effective therapy during the early phase of bilirubin-induced neurologic dysfunction can prevent chronic kernicteric sequelae [10].

Advanced signs of bilirubin encephalopathy are cessation of feeding, bicycling movements, irritability, seizures, fever, and altered mental status. These late findings are predictors of severe kernicteric sequelae. The final stage is chronic irreversible bilirubin encephalopathy, also known as kernicterus [10]. The classic tetrad [11] of chronic irreversible bilirubin encephalopathy are athetoid cerebral palsy, deafness or hearing loss, impairment of upward gaze, and enamel dysplasia of the primary teeth.

Kernicterus was rarely seen in the decades following the introduction of phototherapy and exchange transfusion, but recent reports suggest it is reemerging despite virtual elimination of $\mathrm{Rh}$ disease [4,11]. The majority of cases reported in the last decade have not occurred in infants with $\mathrm{ABO}, \mathrm{Rh}$, other hemolytic disease but in apparently healthy near-term and term infants with significantly elevated bilirubin levels (well above $30 \mathrm{mg} / \mathrm{dL}$ ) [90].

Bhutani and colleagues [6] preformed a review in which steps were designed to facilitate a safer experience with newborn jaundice and prevent the feared manifestation of severe hyperbilirubinemia-kernicterus. A root cause analysis of cases of kernicterus was also performed by the American Academy of Pediatrics Subcommittee on Neonatal Hyperbilirubinemia who identified several potentially correctable factors that are associated with the development of kernicterus [89]. Based on Bhutani's review and the recommendations of the subcommittee, the principles that are most applicable to the practice of emergency medicine include an increased level of concern by the clinician, an objective assessment of the presence or absence of jaundice, the identification of risk factors of potential severe disease, careful attention to parental concern regarding jaundice, the identification of poor feeding and lethargy, and timely intervention for infants with TSB greater than the 95 th percentile $[6,89]$.

\section{Emergency department management and disposition}

Emergency department management of the jaundiced neonate depends on several factors. Management of jaundice in the term newborn is challenging because the clinician must balance the risks of aggressive versus conservative management [70]. Careful consideration must be given to the risks and benefits of each therapeutic intervention. Clinical decision making is aided by weighing variables such as the infant's age, clinical appearance, bilirubin level, the etiology, and the timing of jaundice. 
In unconjugated hyperbilirubinemia, the ultimate goal is the prevention of kernicterus and its potentially devastating effects. The full-term, wellappearing, and afebrile neonate without significant risk factors and a bilirubin level less than $17 \mathrm{mg} / \mathrm{dL}$ may be simply observed, monitoring closely for increasing jaundice. Any dehydration should be corrected according to weight with normal saline boluses, and the frequency of feeding may be increased to aid in the excretion of bilirubin. Frequent feeding and frequent stools aid in bilirubin excretion and should be encouraged. The issue of continuing breastfeeding often arises. The American Academy of Pediatrics does not recommend the discontinuation of breastfeeding in healthy term newborns and encourages frequent breastfeeding of at least eight to ten times per 24-hour time period [1,2]. The major risk of interrupting breastfeeding is that the interruption may result in permanent discontinuation, losing its considerable benefits; however, a temporary cessation may be considered to augment phototherapy [70]. After bilirubin has fallen to a safe level, breastfeeding should be reinstated and the adequacy of this intake monitored to ensure proper hydration. Supplemental feedings in the form of water or dextrose solutions have not been found to precipitate the resolution of serum bilirubin levels to normal, nor are they preventative against the development of jaundice; therefore, they are not routinely recommended for healthy breastfed neonates. Depending on the preferences of the newborn's parents and physician, several options for feeding exist (with or without phototherapy) [2]. A candid conversation should be entered with the newborn's parents outlining the risks and benefits of the continuation of breastfeeding with close monitoring, supplementation with formula, or a brief substitution of breastfeeding with formula, each of which may be implemented with or without phototherapy. Ultimately, feeding options for jaundiced newborns sufficiently healthy to be monitored on an outpatient basis depend on both the parents' and physician's preferences [2]. In cases of breast milk jaundice, one consideration is the temporary cessation of breast milk with reintroduction after the bilirubin has fallen to a safe level.

Phototherapy, one of the mainstays of therapy for the jaundiced neonate, is carried out with a blue light that causes photoconversion of the bilirubin molecule to a water-soluble product that is excreted in the urine and stool. The use of phototherapy may lead to greater water loss; therefore, fluid intake must be increased by $20 \%$. Breastfeeding may be continued. A general rule of thumb regarding when to initiate phototherapy for a full-term healthy infant is to begin the therapy at a bilirubin level of $17 \mathrm{mg} / \mathrm{dL}$ or higher and to discontinue therapy once the bilirubin level is less than $12 \mathrm{mg} / \mathrm{dL}$. Table 2 lists further recommendations based on age in hours and risk. Phototherapy should be started at lower bilirubin levels $(15 \mathrm{mg} / \mathrm{dL})$ for neonates with a rapid rise in bilirubin, when $\mathrm{ABO}$ incompatibility exists, and in the preterm and near-term infant [1].

"Near-term" is a phrase used to describe newborns who do not meet the World Health Organization's definition of 38 to 42 weeks' gestation but are 
Table 2

American Academy of Pediatrics recommendations for phototherapy

\begin{tabular}{|c|c|c|c|c|c|}
\hline \multirow[b]{3}{*}{ Risk category } & \multicolumn{5}{|c|}{ Total serum bilirubin $(\mathrm{mg} / \mathrm{dL})$} \\
\hline & \multicolumn{5}{|c|}{$\overline{\text { Age }(\mathrm{h})}$} \\
\hline & 24 & 36 & 48 & 72 & 96 \\
\hline High risk $\left(35-37\right.$ weeks + risk factors $\left.{ }^{\mathrm{a}}\right)$ & 8 & 9 & 11 & 13 & 14 \\
\hline $\begin{array}{l}\text { Medium risk ( } \geq 38 \text { weeks }+ \text { risk factors or } 35-37 \\
\text { weeks and well) }\end{array}$ & 10 & 12 & 13 & 15 & 17 \\
\hline Low risk ( $>38$ weeks and well) & 12 & 13 & 15 & 18 & 20 \\
\hline
\end{tabular}

over $2500 \mathrm{~g}$ in birth weight $[35,89,91]$. A commentary by the American Academy of Pediatrics Subcommittee on Neonatal Hyperbilirubinemia concluded that newborns who are near-term are at significant risk of severe hyperbilirubinemia [89]. A prospective investigation by Sarici and colleagues [91] determined that neonates between 35 and 37 weeks' gestation (nearterm newborns) were 2.4 times more likely than those at 38 to 42 weeks to develop significant hyperbilirubinemia. These investigators stated nearterm infants should be considered to be a high-risk group because one of four of these infants requires phototherapy for jaundice.

Exchange transfusion should be initiated emergently for markedly elevated serum bilirubin levels (Table 3). A body of literature suggests that exchange transfusion to maintain a bilirubin level below $20 \mathrm{mg} / \mathrm{dL}$ prevents kernicterus in infants with severe hemolytic disease of the newborn $[86,87,92,93]$. An exchange transfusion is recommended for term infants with hemolysis, if phototherapy is unable to maintain the total bilirubin

Table 3

American Academy of Pediatrics recommendations for exchange transfusion

\begin{tabular}{|c|c|c|c|c|c|}
\hline \multirow[b]{3}{*}{ Risk category } & \multicolumn{5}{|c|}{ Total serum bilirubin $(\mathrm{mg} / \mathrm{dL})$} \\
\hline & \multicolumn{5}{|c|}{$\overline{\text { Age (h) }}$} \\
\hline & 24 & 36 & 48 & 72 & 96 \\
\hline High risk $\left(35-37\right.$ weeks + risk factors $\left.{ }^{\mathrm{a}}\right)$ & 15 & 16 & 17 & 18 & 19 \\
\hline $\begin{array}{l}\text { Medium risk ( } \geq 38 \text { weeks }+ \text { risk factors or } 35-37 \\
\text { weeks and well) }\end{array}$ & 16.5 & 18 & 19 & 20 & 22 \\
\hline Low risk ( $>38$ weeks and well) & 19 & 21 & 22 & 24 & 25 \\
\hline
\end{tabular}

${ }^{a}$ Risk factors are defined as isoimmune hemolytic disease, G6PD deficiency, asphyxia, significant lethargy, temperature instability, sepsis, acidosis.

Data from the American Academy of Pediatrics Clinical Practice Guidelines Subcommittee on Hyperbilirubinemia. Management of hyperbilirubinemia in the newborn infant 35 or more weeks of gestation. Pediatrics 2004;114:297-316. 
level below 17.5 to $23.4 \mathrm{mg} / \mathrm{dL}$, or for any neonate showing signs of kernicterus [70]. Newman and colleagues [94] found that when high TSB levels were treated with phototherapy or exchange transfusion, there was not an association with adverse neurodevelopment. An exchange transfusion will reduce the bilirubin concentration by approximately $50 \%$ and should be used with concurrent phototherapy.

Pharmacologic therapy may also be considered in conjunction with a neonatologist. Phenobarbital and ursodeoxycholic acid have been used to improve biliary flow and lower bilirubin levels [35]. Tin mesoporphyrin decreases bilirubin production by inhibiting heme oxygenase and is awaiting US Food and Drug Administration approval [35].

Admission is indicated for all ill-appearing neonates, for those found to be anemic, for bilirubin levels in the range of phototherapy or exchange requirements, and for pathologic or conjugated hyperbilirubinemia [1]. The latter condition always conceals serious underlying disease. Although conjugated bilirubin lacks the neurotoxicity of the unconjugated form, steps should immediately be taken to identify and treat the precipitating disorder. Jaundice is an early indicator of sepsis or urinary tract infection, and if such an infectious etiology is found, antibiotics should be started immediately. The anatomic causes of biliary atresia or obstruction due to a choledochal cyst must be identified early, and surgical evaluation should be prompt [2].

\section{Prognosis}

For the majority of cases, when treated early and appropriately, the prognosis is favorable. This success is evidenced by an investigation by Newman and colleagues [94] who determined that when high TSB levels were treated with phototherapy or exchange transfusion, there was not an association with adverse neurodevelopment.

\section{Summary}

Neonatal hyperbilirubinemia is estimated to occur in the majority of term infants in the first week of life, and approximately $2 \%$ reach a TSB greater than $20 \mathrm{mg} / \mathrm{dL}$ [1-4]. Secondary to early hospital discharge, neonatal jaundice has been transformed from an inpatient issue to an outpatient one that is often dealt with by the emergency physician. Consequently, the emergency physician should be comfortable with the presentation, evaluation, and management of the jaundiced newborn. Kernicterus, a rare but catastrophic complication of neonatal hyperbilirubinemia, is preventable and until recently was thought to be extinct. The role of the emergency physician is to balance the risks and benefits of diagnostic evaluation and management in the care of the jaundiced neonate. 


\section{Acknowledgment}

The authors thank Jeahan R. Hanna, MD, and Elshaday Messele for their support and contributions to this manuscript.

\section{References}

[1] American Academy of Pediatrics, Subcommittee on Neonatal Hyperbilirubinemia. Practice parameter: management of hyperbilirubinemia in the healthy term newborn. Pediatrics 1994; 94:558-5.

[2] Claudius I, Fluharty C, Boles R. The emergency department approach to newborn and childhood metabolic crisis. Emerg Med Clin North Am 2005;23:843-83.

[3] Newman TB, Escobar GJ, Gonzales VM, et al. Frequency of neonatal bilirubin testing and hyperbilirubinemia in a large health maintenance organization. Pediatrics 1999;104(5): 1198-203.

[4] Chou S-C, Palmer RH, Ezhuthachan S, et al. Management of hyperbilirubinemia in newborns: measuring performance by using a benchmarking model. Pediatrics 2003;112(6): 1264-73.

[5] Bhutani VK, Johnson L, Sivieri EM. Predictive ability of a predischarge hour-specific serum bilirubin for subsequent significant hyperbilirubinemia in healthy term and near-term newborns. Pediatrics 1999;103:6-14.

[6] Bhutani VK, Donn SM, Johnson LH. Risk management of severe neonatal hyperbilirubinemia to prevent kernicterus. Clin Perinatol 2005;32(1):125-39.

[7] Eggert LD, Wiedmeiser SE, Willson J, et al. The effect on instituting a prehospital-discharge newborn bilirubin screening program in an 18-hospital health system. Pediatrics 2006;117: $855-62$.

[8] Johnson L, Brown AK. A pilot registry for acute and chronic kernicterus in term and nearterm infants. Pediatrics 1999;104(Suppl 3):736.

[9] Johnson L, Bhutani VK, Brown AK. System-based approach to management of neonatal jaundice and prevention of kernicterus. J Pediatr 2002;140(4):396-403.

[10] Bhutani VK, Johnson LH, Maisels MJ, et al. Kernicterus: epidemiological strategies for its prevention through systems based approaches. J Perinatol 2004;24:650-62.

[11] Shapiro SM. Bilirubin toxicity in the developing nervous system. Pediatr Neurol 2003;29(5): 410-21.

[12] Tiker F, Gulcan H, Kilicdag H, et al. Extreme hyperbilirubinemia in newborn infants. Clin Pediatr (Phila) 2006;45(3):257-61.

[13] Dennery PA, Seidman DS, Stevenson DK. Neonatal hyperbilirubinemia. N Engl J Med 2001;344(8):581-90.

[14] Gartner LM, Catz CS, Yaffe SJ. Neonatal Bilirubin Workshop. Pediatrics 1994;94(4): $537-40$.

[15] Newman TB, Xiong B, Gonzales VM, et al. Prediction and prevention of extreme hyperbilirubinemia in a mature health maintenance organization. Arch Pediatr Adolesc Med 2000; 154:1140-7.

[16] Seidman DS, Stevenson DK, Ergaz Z, et al. Hospital readmission due to neonatal hyperbilirubinemia. Pediatrics 1995;96:727-9.

[17] Maisels MJ, Kring E. Length of stay, jaundice, and hospital readmission. Pediatrics 1998; 101:995-8.

[18] Bhutani VK, Johnson LH, Keren R. Diagnosis and management of hyperbilirubinemia in the term neonate: for a safer first week. Pediatr Clin North Am 2004;51:843-61.

[19] Vales T. Problems with prediction of neonatal hyperbilirubinemia. Pediatrics 2001;108: $175-7$.

[20] Maisels M. Jaundice in the newborn. Pediatr Rev 1982;10:305-19. 
[21] American Academy of Pediatrics Subcommittee on Hyperbilirubinemia. Management of hyperbilirubinemia in the new born infant 35 or more weeks of gestation. Pediatrics 2004; 114:297-316.

[22] Porter LM, Dennis BL. Hyperbilirubinemia in the term newborn. Am Fam Physician 2002; 65(4):599-605.

[23] Kaplan M, Hammerman C. Understanding and preventing severe neonatal hyperbilirubinemia: is bilirubin neurotoxity really a concern in the developed world. Clin Perinatol 2004; 31(3):555-75.

[24] Khoury MJ, Calle EE, Joesoef RM. Recurrence risk of neonatal hyperbilirubinemia in siblings. Am J Dis Child 1988;142(10):1065-9.

[25] Knudsen A, Ebbesen F. Cephalocaudal progression of jaundice in newborns admitted to neonatal intensive care units. Biol Neonate 1997;71:357-61.

[26] Bhutani VK, Gourley GR, Adler S, et al. Noninvasive measurement of total serum bilirubin in a multiracial predischarge newborn population to assess the risk of severe hyperbilirubinemia. Pediatrics 2000;106(2):e17.

[27] Davidson LT, Merritt KK, Weech AA. Hyperbilirubinemia in the newborn. Am J Dis Child 1941;61:958-80.

[28] Moyer VA, Ahn C, Sneed S. Accuracy of clinical judgment in neonatal jaundice. Arch Pediatr Adolesc Med 2000;154:391-4.

[29] Tenovuo A. Neonatal complications in small-for-gestational age neonates. J Perinat Med 1988; 16:197-201.

[30] Keren R, Bhutani VK, Luan X, et al. Identifying newborns at risk of significant hyperbilirubinemia: a comparison of two recommended approaches. Arch Dis Child 2005;90: 415-21.

[31] Thacker KE, Lim T, Drew JH. Cephalhematoma: a 10 year review. Aust N Z J Obstet Gynecol 1987;27(3):210-2.

[32] Perlow JH, Wigton T, Hart J, et al. Birth trauma: a five-year review of incidence and associated perinatal factors. J Reprod Med 1996;41(10):754-60.

[33] Davies DP, Gomersall R, Robertson R, et al. Neonatal jaundice and maternal oxytocin infusion. Br Med J 1973;3:476-7.

[34] Chung MA. Galactosemia in infancy: diagnosis, management, and prognosis. Pediatr Nurs 1997;23(6):563-8.

[35] Maisels M. Neonatal jaundice. Pediatr Rev 2006;27(12):443-54.

[36] Waldron P, De Alarcon P. ABO hemolytic disease of the newborn: a unique constellation of findings in siblings and review of protective mechanisms in the fetal-maternal system. Am J Perinatol 1999;16(8):391-8.

[37] Zawodnik SA, Bonnard GD, Gautier E. Antibody-dependent cell-mediated destruction of human erythrocytes sensitized in ABO and rhesus fetal-maternal incompatibilities. Pediatr Res 1976;10:791-6.

[38] Kaplan M, Hammerman C. Severe neonatal hyperbilirubinemia: a potential complication of glucose-6-phosphate dehydrogenase deficiency. Clin Perinatol 1998;25(3):575-90.

[39] Iranpour R, Akbar MR, Haghshenas I. Glucose-6-phosphate dehydrogenase deficiency in neonates. Indian J Pediatr 2003;70(11):855-7.

[40] Huang MJ, Kua KE, Teng HC, et al. Risk factors for severe hyperbilirubinemia in neonates. Pediatr Res 2004;56(5):682-9.

[41] Atay E, Bozaykut A, Ipek IO. Glucose-6-phosphate dehydrogenase deficiency in neonatal indirect hyperbilirubinemia. J Trop Pediatr 2006;52(1):56-8.

[42] Hammer SG, Lewan RB. Neonatal hyperbilirubinemia caused by pyruvate kinase deficiency. J Am Board Fam Med 1988;1(4):288-90.

[43] Kedar PS, Warang P, Colah RB, et al. Red cell pyruvate kinase deficiency in neonatal jaundice cases in India. Indian J Pediatr 2006;73(11):985-8.

[44] Trucco JI, Brown AK. Neonatal manifestations of hereditary spherocytosis. Am J Dis Child 1967;113:263-70. 
[45] Passi GR, Saran S. Neonatal hyperbilirubinemia due to hereditary spherocytosis. Indian Pediatr 2004;41:199.

[46] Schroter W, Kahsnitz E. Diagnosis of hereditary spherocytosis in newborn infants. J Pediatr 1983;103:460-3.

[47] Laosombat V, Dissaneevate S, Peerapittayamongkol C, et al. Neonatal hyperbilirubinemia associated with southeast Asian ovalocytosis. Am J Hematol 1999;60:136-9.

[48] Austin RF, Desforges JF. Hereditary elliptocytosis: an unusual presentation of hemolysis in the newborn associated with transient morphologic abnormalities. Pediatrics 1969;44(2): 196-200.

[49] Gartner LM, Herschel M. Jaundice and breastfeeding. Pediatr Clin North Am 2001;48(2): 389-99.

[50] Jansen PLM. Diagnosis and management of Crigler-Najjar syndrome. Eur J Pediatr 1999; 158(Suppl 2):S89-94.

[51] Strauss KA, Robinson DL, Vreman HJ, et al. Management of hyperbilirubinemia and prevention of kernicterus in 20 patients with Crigler-Najjar disease. Eur J Pediatr 2006;165(5): $306-19$.

[52] LaFranchi SH, Murphey WH, Foley TP, et al. Neonatal hypothyroidism detected by the northwest regional screening program. Pediatrics 1979;63(2):180-91.

[53] Schneider AP. Breast milk jaundice in the newborn: a real entity. JAMA 1986;255(23): $3270-4$.

[54] Kivlahan C, James EJ. The natural history of neonatal jaundice. Pediatrics 1984;74(3): 364-70.

[55] Brown LP, Arnold A, Allison D, et al. Incidence and pattern of jaundice in healthy breast-fed infants during the first month of life. Nurs Res 1993;42(2):106-10.

[56] Osborn LM, Reiff MI, Bolus R. Jaundice in the full-term neonate. Pediatrics 1984;73(4): $520-5$.

[57] Poland RL. Breast-milk jaundice. J Pediatr 1981;99(1):86-8.

[58] Gerardi M. Neonatal emergencies: fever, jaundice, respiratory distress, cyanotic heart disease, and behavioral complaints. Pediatric Emergency Medicine Reports 1996;1(12):113-24.

[59] Brown AK. Neonatal jaundice. Pediatr Clin North Am 1962;9(3):575-603.

[60] Bernstein J, Brown AK. Sepsis and jaundice in early infancy. Pediatrics 1962;29:873-82.

[61] Hamilton JR, Sass-Kortsak A. Jaundice associated with severe bacterial infection in young infants. J Pediatr 1963;63:121-32.

[62] Arthur AB, Wilson BR. Urinary infection presenting with jaundice. Br Med J 1967;1:539-40.

[63] Rooney JC, Hill DJ, Danks DM. Jaundice associated with bacterial infection in the newborn. Am J Dis Child 1971;122(1):39-41.

[64] Littlewood JM. 66 Infants with urinary tract infections in first month of life. Arch Dis Child 1972;47:218-26.

[65] Seeler RA. Urosepsis with jaundice due to hemolytic Escherichia coli. Am J Dis Child 1973; 126:414.

[66] Linder N, Yatsiv I, Tsur M, et al. Unexplained neonatal jaundice as an early diagnostic sign of septicemia in the newborn. J Perinatol 1988;8(4):325-7.

[67] Escobedo MB, Barton LL, Marshall RE, et al. The frequency of jaundice in neonatal bacterial infection. Clin Pediatr 1974;13(8):656-7.

[68] Chavalitdhamrong PO, Escobedo MB, Barton LL, et al. Hyperbilirubinemia and bacterial infection in the newborn. Arch Dis Child 1975;50(8):652-4.

[69] Maisels MJ, Kring E. Risk of sepsis in newborns with severe hyperbilirubinemia. Pediatrics 1992;90(5):741-3.

[70] Newman TB, Maisels JM. Evaluation and treatment of jaundice in the term newborn: a kinder, gentler approach. Pediatrics 1992;89:809-18.

[71] Schreiner RL, Glick MR. Interlaboratory bilirubin variability. Pediatrics 1982;69:277-81.

[72] Watkinison LR, St John A, Penberthy LA. Investigation into paediatric bilirubin analyses in Australia and New Zealand. J Clin Pathol 1982;35:52-8. 
[73] Chan KM, Scott MG, Wu TW, et al. Inaccurate values for direct bilirubin with some commonly used direct bilirubin procedures. Clin Chem 1985;31:1560-3.

[74] Rosenthal P. The laboratory method as a variable in the diagnosis of hyperbilirubinemia. Am J Dis Child 1987;141(10):1066-8.

[75] Newman TB, Hope S, Stevenson D. Direct bilirubin measurements in jaundiced term newborns: a re-evaluation. Am J Dis Child 1991;145:1305-9.

[76] LO SF, Doumas TB, Ashwood ER. Performance of bilirubin determinations in US laboratories-revisited. Clin Chem 2004;51(1):190-4.

[77] Newman TB, Liljestrand PJ, Escobar GJ. Hyperbilirubinemia benchmarking. Pediatrics 2004;114(1):323.

[78] Vreman HJ, Verter J, Oh W, et al. Interlaboratory variability of bilirubin measurements. Clin Chem 1995;42:869-73.

[79] Seeler RA, Hahn K. Jaundice in urinary tract infection in infancy. Am J Dis Child 1969;18: 553-8.

[80] Ng SH, Rawstrong JR. Urinary tract infections presenting with jaundice. Arch Dis Child 1971;46:173-6.

[81] Seeler R. Hemolysis due to gram-negative urinary tract infection. Birth Defects Orig Artic Ser 1977;13:425-31.

[82] Garcia FJ, Nager AL. Jaundice as an early sign of urinary tract infection in infancy. Pediatrics 2002;109(5):846-51.

[83] MacDonald MG. Hidden risks: early discharge and bilirubin toxicity due to glucose 6-phosphate dehydrogenase deficiency. Pediatrics 1995;96(4):734-8.

[84] Van Praagh R. Diagnosis of kernicterus in the neonatal period. Pediatrics 1961;28:870-6.

[85] Connolly AM, Volpe JJ. Clinical feature of bilirubin encephalopathy. Clin Perinatal 1990; 17:371-9.

[86] Mollison Pl, Cutbush M. Haemolytic disease of the newborn. Recent Advances in Pediatrics $1954 ; 110$.

[87] Hsia Dy-Y, Allen FH, Gellits SS, et al. Erythroblastosis fetalis. VIII. Studies of serum bilirubin in relation to kernicterus. N Engl J Med 1952;247:668-71.

[88] Volpe J. Bilirubin and brain injury. Neurology of the newborn. 4th edition. Philadelphia: WB Saunders; 2001. p. 521-46.

[89] American Academy of Pediatrics Subcommittee on Neonatal Hyperbilirubinemia. Neonatal jaundice and kernicterus. Pediatrics 2001;108:763-5.

[90] Maisels MJ, Newman TB. Predicting hyperbilirubinemia in newborns: the importance of timing. Pediatrics 1999;103:493-4.

[91] Sarici SU, Serdar MA, Korkmaz A, et al. Incidence, course and prediction of hyperbilirubinemia in near-term and term newborns. Pediatrics 2004;113(4):775-80.

[92] Mollison PL, Walker W. Controlled trials of the treatment of haemolytic disease of the newborn. Lancet 1952;1:429-33.

[93] Ellis MI, Hey EN, Walker W. Neonatal death in babies with rhesus isoimmunization. Q J Med 1979;48:211-25.

[94] Newman, Thomas B, Liljestrand P, et al. The Jaundice and Infant Feeding Study Team. Outcomes among newborns with total serum bilirubin levels of $25 \mathrm{mg}$ per deciliter or more. N Engl J Med 2006;354(18):1889-900. 